\title{
Post-emetic oesophageal rupture - successful conservative management
}

S K Misser, MB ChB, FCRad (D)
J Maharajh, MB ChB, MMed, FFRad (D)
$\begin{gathered}\text { Department of Radiology, Nelson R Mandela School of Medicine, King Edward } \\ \text { VIII Hospital, Durban }\end{gathered}$
H T Campbell, MB ChB, FFRad (D)
Department of Radiology, R K Khan Hospital, Durban
M Naidoo, MB ChB, FCS (SA)
A A Gounden, MB ChB, FCS (SA)
Department of Surgery, R K Khan Hospital, Durban

Boerhaave's syndrome, also known as spontaneous oesophageal rupture or post-emetic oesophageal rupture (PEOR), is perhaps one of the more challenging clinical syndromes encountered in general surgical practice. Co-morbidity, delayed diagnosis and associated delayed institution of therapy have resulted in an almost universally poor prognosis, even in specialist surgical units. Surgical intervention is usually the mainstay of therapy. There have been few reported cases of successful non-operative management. Here we report one such success story.

\section{Case history}

A 57-year-old man presented with severe epigastric pain, excessive vomiting and four episodes of frank haematemesis. Prior to the onset of the vomiting, he had experienced dull abdominal pain, which later localised to the left lower quadrant. Of note, the patient had a background history of sigmoid diverticulosis.

The chest radiograph demonstrated a pneumomediastinum and bilateral pleural effusions, larger on the left. In light of the severe haematemesis, an urgent upper endoscopy was performed. Two distinct perforations were identified in the distal oesophagus, approximately 2.5 $\mathrm{cm}$ from the oesophago-gastric junction. Gastritis was also present, and a solitary ulcer was noted on the lesser curve of the stomach.

CT scan of the chest and abdomen (Fig. 1) confirmed perforation of the distal oesophagus, with associated pneumomediastinum and bilateral pleural effusions. Deep in the left iliac fossa, sigmoid diverticulae were demonstrated, together with a contiguous 3.5 by $4 \mathrm{~cm}$ abscess (Fig. 2).

A water-soluble contrast swallow (Fig. 3) revealed oesophageal rupture with focal contrast extravasation. The diagnosis was clear. The patient had developed sigmoid diverticulitis and an associated abscess, which resulted in significant vomiting and spontaneous oesophageal rupture or Boerhaave's syndrome.

After discussion with the patient and his family, a conservative approach was chosen. Follow-up water-soluble contrast swallows were performed at weekly intervals. By six weeks, the patient was well with no residual leak or clinical complaints.

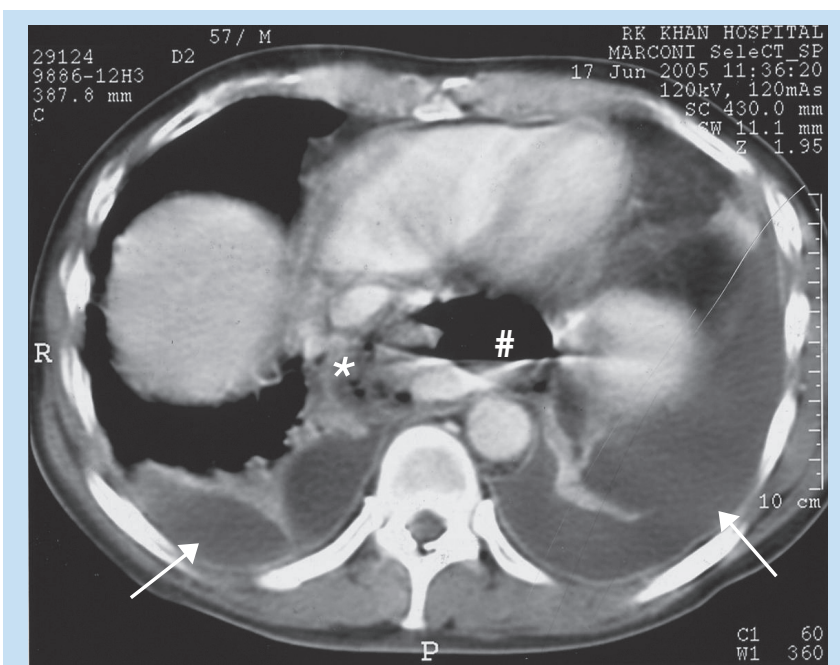

Fig. 1. Chest CT scan at lower thoracic level reveals bilateral pleural collections $(\rightarrow)$, pneumomediastinum $\left({ }^{*}\right)$ and peri-oesophageal swelling with air-fluid level (\#).

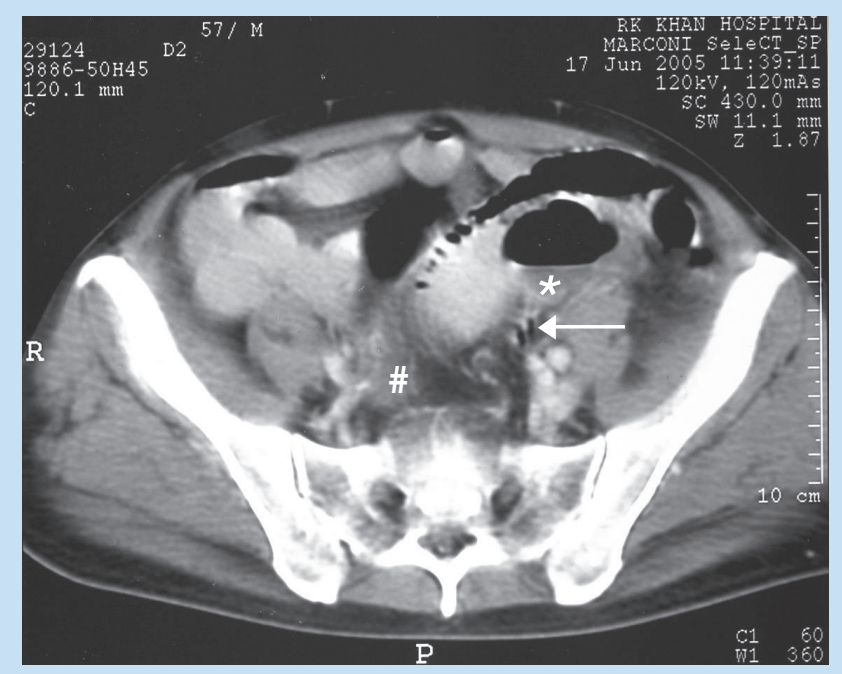

Fig. 2. CT scan through pelvis demonstrates sigmoid diverticulae $(\rightarrow)$, mesocolonic stranding (\#) and an interloop abscess (*).

\section{Discussion}

Boerhaave's syndrome, also known as post-emetic oesophageal rupture (PEOR), is one of the more challenging clinical syndromes encountered in general surgical practice. Co-morbidity, delayed diagnosis, and associated delayed institution of therapy have resulted in an almost universally poor prognosis, even in specialist units. Of all gastro-intestinal perforations, PEOR is the most frequently fatal. ${ }^{1} \mathrm{~A}$ keen index of clinical suspicion, appropriate immediate investigations and timeous referral to the surgical team improves outcome. Untreated, PEOR has a nearly $100 \%$ mortality rate. ${ }^{2,3}$ 


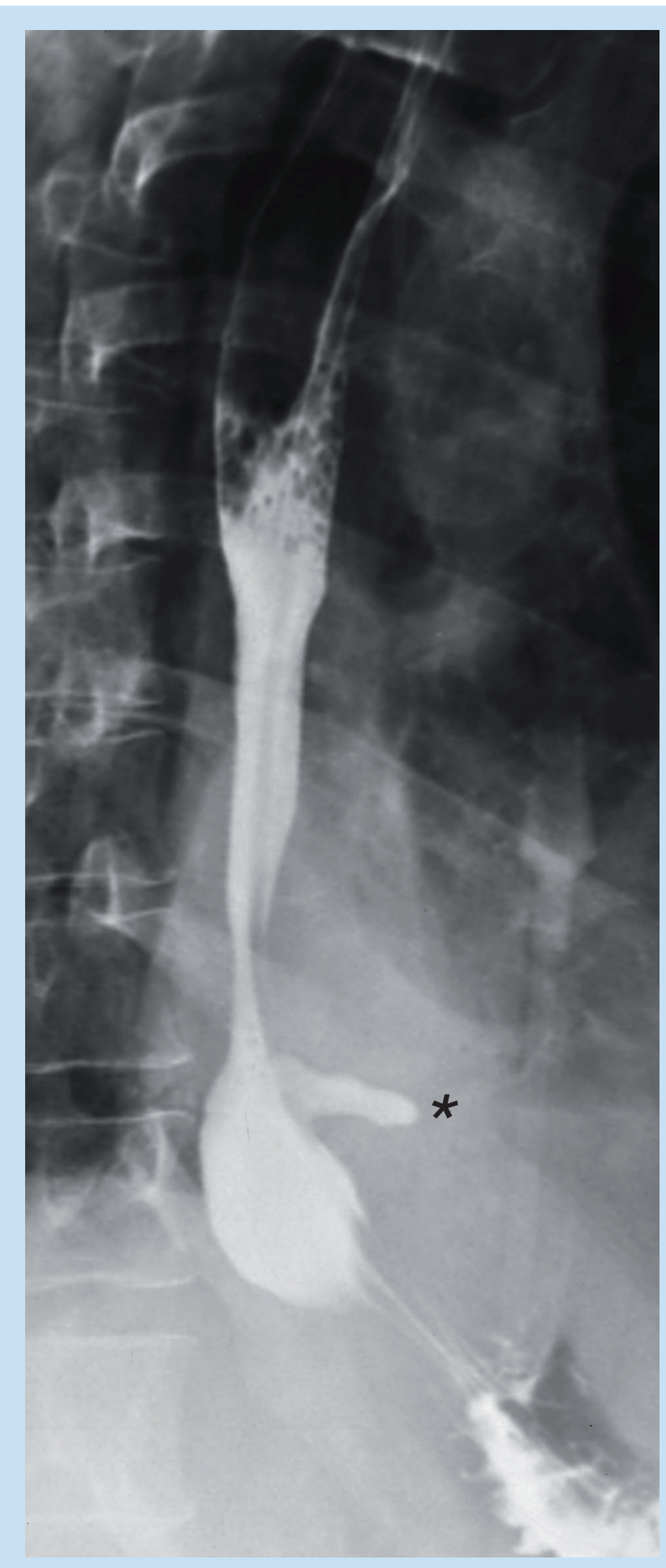

Fig. 3. Contrast swallow demonstrating free distal dorsal oesophageal perforation $\left(^{*}\right)$ along the left lateral wall.

Radiology plays a pivotal role in the diagnosis of PEOR. The chest radiograph may demonstrate a pneumomediastinum, pleural effusions (especially left-sided) or hydropneumothorax in PEOR. Mediastinal widening, air-fluid levels in the mediastinum and subcutaneous emphysema are important ancillary findings. The V-sign of Naclerio is occasionally identified as a V-shaped lucency projected through the cardiac shadow, representing air loculated between the left hemi-diaphragm and the left inferior mediastinal border. A negative or normal chest radiograph does not exclude PEOR. Han et al. ${ }^{4}$ documented normal chest radiography in $12 \%$ of patients with PEOR.

Water-soluble contrast oesophagography is the next investigation of choice to delineate oesophageal rupture. Some authors advise using dilute barium and decubitus or shoot-through positioning if the initial study fails to reveal any perforation. ${ }^{5}$ Contrast swallow studies are a useful tool for follow-up of these patients in the subsequent weeks of convalescence.

CT scanning expedites the initial assessment of PEOR, especially in patients who are too ill to undergo endoscopy or contrast studies. CT is capable of demonstrating minor leaks into the posterior mediastinum. An added advantage CT has over endoscopy or a contrast swallow is its ability to demonstrate extra-luminal detail.

Generally, most patients with PEOR are best served by some form of surgical oesophageal repair. ${ }^{2}$ The place for oesophageal stenting in PEOR has not been clearly established, despite some reports of success. ${ }^{6,7}$ The conservative, non-surgical management of proven oesophageal disruption is limited to those patients with a well-contained leak, no pleural soiling and no clinically evident sepsis. These patients are usually stable and present later. Very few patients with mediastinitis, pleural contamination and incipient multi-organ dysfunction survive with a non-operative management regimen. Those who do (as in the index patient), are usually worth reporting.

1. Singh H, Warshawsky ME, Herman S, Shanies HM. Spontaneous oesophageal rupture: Boerhaave's Syndrome. Clinical Pulmonary Medicine 2003; 10: 177-182.

2. Vial CM, Whyte RI. Boerhaave’s Syndrome: Diagnosis and treatment. Surg Clin North Am 2005; 85: 515-524.

3. Pate JW, Walker WA, Cole FH Jr, Owen EW, Johnson WH. Spontaneous rupture of the oesophagus: a 30year experience. Ann Thorac Surg 1989; 47: 689-692

4. Han SY, McElvein RB, Aldrete JS, Tishler JM. Perforation of the oesophagus: correlation of site and cause with plain film findings. Am J Roentgenol 1985; 145: 537-540.

5. Freeman A. In: Grainger RG, Allison DJ, eds. Grainger and Allison's Diagnostic Radiology - A Textbook of Medical Imaging. 4th ed. London: Churchill Livingstone, 2001: 1033.

6. Adam A, Watkinson AF, Dussek J. Boerhaave syndrome: To treat or not to treat by means of insertion of a metallic stent. J Vasc Interv Radiol 1995; 6: 741-743; Discussion 744-746.

7. Neff C, Lawson DW. Boerhaave’s syndrome: Interventional radiologic management. Am J Roentgenol 1985; 145: 819-820. 